\title{
Innovación educativa y nuevas tecnologías aplicadas a la enseñanza
}

Algunos proyectos y experiencias metodológicas

\section{Xandra Santos Palmou}

\section{(2) OpenEdition}

\section{Journals}

Edición electrónica

URL: https://journals.openedition.org/cher/948

DOI: $10.4000 /$ cher. 948

ISSN: 2803-5992

\section{Editor}

Presses universitaires de Strasbourg

Edición impresa

Fecha de publicación: 12 diciembre 2019

Paginación: 129-139

ISBN: 979-10-344-0057-7

ISSN: 1968-035X

\section{Referencia electrónica}

Xandra Santos Palmou, «Innovación educativa y nuevas tecnologías aplicadas a la enseñanza»,

reCHERches [En línea], 23 | 2019, Publicado el 27 septiembre 2021, consultado el 19 noviembre 2021. URL: http://journals.openedition.org/cher/948 ; DOI: https://doi.org/10.4000/cher.948 


\title{
Innovación educativa y nuevas tecnologías aplicadas a la enseñanza Algunos proyectos y experiencias metodológicas
}

\author{
Xandra Santos Palmou 1
}

\begin{abstract}
A lo largo de estas líneas expondremos algunas propuestas y experiencias docentes llevadas a cabo en el ámbito universitario francés, concretamente en la Facultad de Lenguas de la Universidad de Estrasburgo. Para ello, nos centramos en un nuevo dispositivo de formación puesto en práctica en las aulas de español como lengua extranjera para los alumnos de LANSAD (Lenguas para Especialistas de otras disciplinas, también llamado español para no especialistas), así como algunas metodologías que hemos llevado a cabo en la asignatura de Historia de España, con el alumnado de primer año de la licenciatura de LLCER (Lenguas, Literaturas y Civilizaciones Extranjeras y Regionales) de la especialidad de español.

Presentamos ambas iniciativas en un único trabajo porque las dos persiguen los mismos objetivos y nos conducen a idénticas reflexiones pedagógicas, pero también porque, como veremos, su comparación nos permite observar que los cambios que se han producido en el aprendizaje de las lenguas extranjeras y en las metodologías docentes van al encuentro de aquellas innovaciones pedagógicas que también se intentan promocionar en el contexto universitario francés $y$, en general, europeo. Aunque no nos centraremos en el ámbito educativo de primaria y secundaria, apuntaremos algunas ideas para mostrar cómo las bases pedagógicas que sostienen los cambios producidos en la enseñanza de lenguas a extranjeros y las que se promueven desde hace ya varios años en el sistema educativo universitario también son convergentes, ya que, en todos estos casos, estas transformaciones responden, por una parte, a un intento de adaptación a los cambios sociales que se han producido en las últimas décadas y, por otro, a una nueva forma común de entender los procesos de enseñanza-aprendizaje. Un aspecto que subyace bajo ambas propuestas es su intento de reflexionar sobre cómo afrontar los retos y dificultades docentes, apostando por la introducción de metodologías que, en los últimos años, se han popularizado gracias a la
\end{abstract}

1 Xandra Santos Palmou, Université des Antilles, ORCID:0000-0001-5161-692X 
integración de las Tecnologías de la Información y de la Comunicación para la Enseñanza (TICE). De este modo, exploraremos los desafíos que surgen a raíz del uso de estos nuevos escenarios de aprendizaje que, apoyándose en las tecnologías, cambian por completo la combinación de los pares espacio/tiempo y presencia/distancia, cuestionándonos sobre su necesidad, sus ventajas y sus inconvenientes.

\section{Contexto social y educativo: cambios socioeconómicos producidos en las últimas décadas y sus implicaciones docentes}

Las transformaciones sociales y económicas que tuvieron lugar a finales del siglo xx y principios del xxi han afectado de lleno a la concepción del sistema de educación superior y a la función social que este debe cumplir. Los resultados se manifiestan en la revisión y renovación de los enfoques educativos, de las metodologías docentes y de los roles que desempeñan el profesorado y el alumnado en el proceso de enseñanza-aprendizaje. Este nuevo escenario socioeconómico también ha tenido como consecuencia un cambio en el papel que otorgamos a las lenguas extranjeras en nuestra sociedad y, por lo tanto, en nuestro sistema educativo. A ello debemos sumar la evolución conocida por las generaciones a las que se forma en la actualidad, por lo que parece evidente que la formación que reciben deba adaptarse a los nuevos destinatarios y a la nueva realidad.

Desde finales del segundo milenio nos venimos refiriendo a la sociedad moderna como la «Sociedad de la Información», la «Sociedad del Conocimiento» o la «Sociedad del Aprendizaje». Tres expresiones que revelan algunas de las más importantes transformaciones que han ocurrido en estos últimos años y que, sin duda, van a seguir produciéndose en los que están por venir. Así, mientras que la base del antiguo sistema económico tradicional era el capital físico o tangible, en el sistema económico actual se pone el énfasis en aspectos vinculados con los procesos de desarrollo intelectual, como son los relativos al conocimiento y la información (Bueno 2010). Es decir, hemos incorporado estos dos conceptos a los procesos económicos como bienes no materiales o intangibles, que, en la actualidad, representan los factores más valiosos. Esta incorporación del conocimiento requiere de una determinada tecnología. Tenemos, por lo tanto, una situación donde el mayor protagonismo lo tiene el manejo de la información, debido a la importancia de las tecnologías de la información y al avance de las plataformas digitales y sus redes de comunicaciones. Si anteriormente se había producido una globalización económica, estos nuevos soportes facilitan que las personas, prácticamente sin barreras físicas ni temporales, puedan comunicarse y transmitir conocimientos o información. Es precisamente en esto en lo que radica la gran revolución de la Sociedad de la Información, ya que estos nuevos soportes y posibilidades nos acercan al conocimiento, a las fuentes del saber, modifican nuestra manera de entender el mundo y alteran nuestro comportamiento, hasta tal punto, que transforman y mejoran también 
nuestra capacidad de aprender. Así, los nuevos soportes redefinen los conceptos tradicionales de conocimiento y de aprendizaje, que ya no se relacionan del mismo modo. De hecho, en el mundo en que vivimos hoy, el recurso más valioso es el conocimiento, lo que implica el reto de aprender a aprender, de saber cómo incorporarlo de una forma continua para promocionar la formación a lo largo de la vida y permitir una mayor autonomía en este proceso de aprendizaje.

De la mano de estas mudanzas socioeconómicas, observamos también una serie de cambios generacionales que debemos destacar. Sin profundizar en cuestiones terminológicas o definitorias sobre la generación de estudiantes que llegan en este momento a las aulas universitarias, y teniendo en cuenta que la validez de las generalizaciones es siempre limitada, no podemos obviar que existen algunas cualidades que suelen manifestarse en muchos de los aprendientes ${ }^{2}$. Nos referimos, por ejemplo, a su capacidad multitarea y a la preferencia por el uso de la tecnología y las redes sociales, o a su dificultad para concentrarse durante periodos de tiempo relativamente largos. Por otro lado, como hijos del mundo globalizado que les ha tocado vivir, están más abiertos a los cambios y más acostumbrados a viajar y a la diversidad cultural. Suelen definirse como menos conformistas, jóvenes para los cuales la comodidad ha dado paso a una mayor habilidad para aceptar nuevos retos y a la búsqueda del reconocimiento. Otras características que se les suele atribuir son sus ansias para desarrollar sus propias ideas y proyectos, y una mayor facilidad para el emprendimiento, es decir, hacen gala de una mayor creatividad y buscan más autonomía en el trabajo. Sin embargo, son una generación que requiere una definición clara de los objetivos y se sienten más motivados cuando estos están en consonancia con sus intereses y persiguen fines prácticos.

Sin duda, los aspectos de mayor importancia de todo lo expuesto anteriormente son aquellos que se reflejan en el ámbito educativo y que tienen implicaciones directas en cómo se integran y qué buscan estos miembros de la Sociedad del Conocimiento y de la Información cuando acceden al sistema de educación superior. En este sentido, y en relación con los Millennials, es decir, aquellos nacidos en la década de 1980 y 1990, quizás una de sus características principales es que «piden cambios y cuestionan la Escuela tradicional, debido al cambio del mercado laboral» (Díaz, López y Roncallo 2017: 199).

Del mismo modo, si observamos las transformaciones que se han promovido en las dos últimas décadas por parte de los diferentes gobiernos en lo que se ha venido denominando el Espacio Europeo de Educación Superior (EEES), se

2 Para acceder a un estudio más profundo sobre el concepto y el término de "generación» y una clasificación de los diferentes grupos generacionales que han existido en la sociedad, de acuerdo con la fecha en que emergen y en la que son relevados por la siguiente generación, puede consultarse el trabajo de Díaz-Sarmiento, López-Lambraño y Roncallo-Lafont 2017. En él se incluye también una perspectiva resumida de las generaciones Baby Boomer, Generación X y Millennials, haciendo énfasis en aspectos como su actitud hacia el trabajo, las principales motivaciones $y$, sobre todo, sus preferencias en educación. 
puede ver que el cambio de paradigma que se viene produciendo responde a los mismos criterios. Conscientes de las evoluciones sociales y económicas que hemos vivido y de que las universidades ocupan un papel central en el desarrollo de esta sociedad del conocimiento actual, las instituciones de enseñanza superior están también inmersas en un proceso de revisión y renovación que tuvo su punto de partida en el año 1999, con la Declaración de Bolonia, y que, hoy en día, todavía está en marcha, como así lo expone Montero Curiel en un trabajo cuyo objetivo es exponer estos cambios y sus consecuencias a nivel educativo:

La construcción del Espacio Europeo de Educación Superior (EEES) supone una magnífica oportunidad para que las universidades aborden un proceso de reforma que les permita adaptarse a la nueva realidad social que nos ha correspondido vivir, la denominada Sociedad del Conocimiento o Sociedad del Saber, sintagma que traduce el inglés knowledge society para referirse a la sociedad actual, producto de las enormes transformaciones que se están produciendo en el mundo moderno. (2010: 21)

En este sentido, las tendencias que se delimitan son claras, debemos pensar en un espacio común europeo, organizado bajo los mismos principios y que potencie una transformación que afecta de lleno a la concepción de la educación superior. En sintonía con la tendencia a una mayor movilidad de los ciudadanos entre los países comunitarios, se pone de manifiesto la necesidad de fomentar nuevos principios y promover un espacio común y homogéneo de educación superior. Esta dimensión europea supone la coordinación entre los países comunitarios para establecer las bases de un marco común de educación, que promueva la movilidad, establezca los mismos principios pedagógicos y facilite la equivalencia de diplomas.

Al mismo tiempo, las universidades del siglo xxi deben responder de manera eficaz a las necesidades generadas por una sociedad posindustrial, globalizada y en la que las nuevas tecnologías de la información ganan cada vez más importancia, ofreciendo a sus estudiantes una formación que sea acorde con aquello que la sociedad les va a demandar cuando comiencen su vida laboral. Las implicaciones pedagógicas son numerosas y los cambios que esto conlleva afectan a varios aspectos, desde una nueva metodología docente, a las transformaciones a la hora de definir los objetivos y de establecer la evaluación, pasando por el papel que ocupan los docentes y los discentes; hasta la forma de entender el propio proceso de «enseñanza-aprendizaje» en el que el segundo elemento del binomio cobra protagonismo.

Por otro lado, si ampliamos nuestra perspectiva, resulta interesante establecer una comparación entre estos procesos de transformación y adaptación de las universidades que hemos comentado con lo ocurrido en el ámbito de la enseñanza de lenguas extranjeras, en concreto desde que en el año 2001 se publicó el Marco común europeo de referencia para las lenguas (MCER 2001). Un documento que, desde entonces, se ha convertido en imprescindible como guía para la enseñanza y aprendizaje de lenguas, así como para su evaluación. Al igual que apuntamos anteriormente para el sistema universitario, este texto realizaba 
un diagnóstico de los cambios sociales que se habían producido, anunciaba la importancia cobrada por las lenguas extranjeras en la sociedad, en concreto, en el marco de los países comunitarios y establecía un cambio de paradigma fundamental para la enseñanza-aprendizaje de las lenguas. En general, podemos afirmar que los cambios que se promovieron en el ámbito universitario europeo y aquellos que se preconizaron para la enseñanza de lenguas, no solo nacen en una misma época, sino que también beben de un paradigma casi idéntico, comparten los mismos enfoques educativos y persiguen objetivos muy similares. En concreto, uno de los aspectos fundamentales que promueven los textos oficiales de ambos sectores es el enfoque basado en competencias, pilar básico en el proceso de enseñanza-aprendizaje. Esta es una definición de la educación que ultrapasa la aproximación por contenidos y se pregunta «qué podemos hacer» con los conocimientos que adquirimos y si somos capaces de aplicarlos para una finalidad concreta en contextos reales.

De hecho, incluso podríamos ampliar nuestro punto de mira y aplicar estas afirmaciones a la enseñanza primaria y secundaria francesa, para la que se promulgó la Ley del 11 de febrero de la Educación Nacional, reformada por la Ley del 8 de julio de 2013 para la refundación de la Escuela, ambas concretadas por el Socle commun de connaissance de compétences (2006) y el Socle Commun de Connaissances de Compétences et de Culture (2015), respectivamente. En este último documento, por ejemplo, se establece una definición de los conocimientos y las competencias basados en cinco dominios, una nueva perspectiva que pretende dejar atrás las tradicionales «disciplinas» o «asignaturas», en consonancia con lo que habíamos apuntado para la enseñanza superior y la adquisición de lenguas extranjeras.

En el caso de la enseñanza de lenguas, el MCER propuso una nueva visión más amplia del alumno, ya que representó al estudiante/usuario de lenguas como un «actor social» que actúa en el entorno social y desempeña un papel en el proceso de aprendizaje. Esto significa un cambio de paradigma tanto en la planificación de los cursos como en la enseñanza, fomentando la participación y la autonomía de los alumnos.

En cuanto al concepto de competencia, este se desarrolló unido al enfoque orientado a la acción, gracias a descriptores del tipo "poder hacer», como así lo refleja el volumen complementario recientemente publicado por el Consejo de Europa al establecer los elementos claves del MCER para la enseñanza y el aprendizaje:

Avec la perspective actionnelle du CECR, on passe des programmes fondés sur une progression linéaire à partir des structures du langage ou d'un ensemble de notions et fonctions prédéterminées, à des programmes fondés sur des analyses de besoins, des tâches de la vie réelle bâties sur des notions et des fonctions choisies délibérément. On favorise ainsi une perspective de compétence illustrée par des descripteurs commençant par 'Je peux (faire)'. L'idée est de concevoir des programmes et des cours fondés sur des besoins de communication dans le monde réel, organisés autour de tâches de la vie réelle à l'aide de descripteurs 'Je 
peux (faire)' qui représentent des objectifs pour l'apprenant. (Consejo de Europa 2018: 26).

Este nuevo volumen actualiza las escalas publicadas por el MCER, pero también profundiza en algunos aspectos que se consideran fundamentales, como son la competencia plurilingüe y pluricultural, y establece como actividades de la lengua la recepción, la producción, la mediación ${ }^{3}$ y la interacción, reconociendo la naturaleza social del aprendizaje y del uso de la lengua. Además, especifica que, en un enfoque orientado a la acción, las tareas colaborativas en una clase de lengua son indispensables, lo que significa que nuestros estudiantes van a «aprender haciendo» (Consejo de Europa 2018: 30).

Si nos centramos en la enseñanza universitaria, las competencias se dividen en competencias generales, aquellas comunes a todas las licenciaturas, y específicas, las que son específicas para cada carrera y que se exigirán para el desempeño específico de cada profesión. Entre estas últimas se establecen tres tipos": las competencias disciplinares (saber), las procedimentales o instrumentales (saber hacer) y las actitudinales (ser). Igualmente, el enfoque orientado a la acción, o lo que hemos denominado "aprender haciendo», encuentra en el ámbito de la enseñanza superior una gran relevancia, ya que para adquirir las competencias que hemos determinado previamente no solo hay que relacionarlas con los contenidos concretos que vamos a trabajar, sino, sobre todo, ponerlas en práctica mediante actividades de aprendizaje y tareas que así lo posibiliten. Estas actividades pueden ser de diverso tipo, desde exposiciones orales, a prácticas individuales, trabajos en equipo o resoluciones de casos, etc. Con ellas, debemos familiarizar a los estudiantes con nuevas acciones, que no se reducen a comprender, saber o memorizar, sino que los llevarán a analizar, aplicar, comunicar, desarrollar, diseñar, resolver, investigar, interpretar, razonar, etc.

En un modelo de enseñanza tradicional, la secuencia seguida para la planificación educativa podría ser:

1. Establecer los conocimientos fundamentales;

2. Impartirlos;

3. Evaluar si los estudiantes los han adquirido; y

3 Para profundizar sobre el concepto de la mediadición en el MCER puede consultarse el trabajo de Cores Bilbao (2017).

4 Algunos ejemplos de estas competencias son: capacidad de análisis y de síntesis; planificación y gestión del tiempo; dominio de las TIC; habilidad para buscar y seleccionar adecuadamente en las fuentes de documentación, tanto por medio de los recursos bibliográficos en soporte tradicional (libros, revistas, etc.), como a través del material en Red (Internet, revistas digitales, Webs, etc.); capacidad para aprender de forma autónoma y suficientemente, con el objeto de seguir formándose a lo largo de toda la vida laboral (formación continua); capacidad de reflexionar de manera crítica y personal, incluyendo toma de decisiones que coadyuven a la resolución de problemas; capacidad de trabajar en equipo; desarrollo de la capacidad de liderazgo; habilidad para trabajar de forma autónoma; etc. (Montero Curiel 2010). 
4. Confiar en que la adquisición de dichos conocimientos los lleve a ser buenos profesionales.

Por el contrario, en los modelos basados en competencias, la secuencia es diferente:

1. Definir las características de un buen profesional (es decir, las competencias o los resultados a los que va dirigido el aprendizaje);

2. Diseñar criterios y estándares para determinar cómo evaluar las competencias;

3. Diseñar actividades y oportunidades de aprendizaje; y

4. Evaluar su adquisición. En esta nueva perspectiva, el papel del estudiante cobra un significado especial, puesto que él mismo debe ser el motor del aprendizaje, mientras que la función del docente se parece más a la de un guía.

Del mismo modo, estos nuevos modelos posibilitan que el alumnado pueda desarrollar una mayor autonomía en el aprendizaje y permiten en mayor medida la adaptación a los diferentes estilos y ritmos de aprendizaje.

\section{Experiencias docentes}

En consonancia con lo expuesto anteriormente, en el siguiente apartado describimos brevemente dos experiencias docentes llevadas a cabo en el seno de la Facultad de Lenguas de la Universidad de Estrasburgo y en el centro de aprendizaje de idiomas SPIRAL. Ambas iniciativas suponen un intento de afrontar algunos retos a los que se enfrentan las universidades francesas en la actualidad, poniendo en práctica los presupuestos y argumentos manifestados más arriba. Desde las instituciones universitarias se potencia el uso de las nuevas plataformas digitales aplicadas a la enseñanza y, en general, el uso de las TICE para complementar las sesiones presenciales. Se ofrecen como herramientas que permiten a las universidades optimizar recursos. Y cada vez más, estas plataformas se presentan como una posible solución al aumento del número de estudiantes y a las dificultades para compatibilizar horarios y espacios por parte de la comunidad educativa, gracias a que permiten poner en marcha dispositivos de enseñanza semipresencial o a distancia.

\section{Espagnol: Autoformation accompagnée}

La primera experiencia docente que describimos se desarrolló en el ámbito de la asignatura de LANSAD, ofrecida por la Facultad de Lenguas al alumnado no hispanista de la Universidad de Estrasburgo. Se trata de una disciplina de español dispensada por el Instituto de Estudios Románicos en el curso 20182019. El diseño de esta asignatura de ELE se llevó a cabo en colaboración con el centro de idiomas SPIRAL, de esta misma universidad. Se optó así por un dispositivo piloto, en el caso del español, de enseñanza semipresencial solo para el nivel A1, es decir, nivel inicial absoluto. El motivo de poner en práctica este dispositivo es el gran número de alumnos que, cada vez más, demandan 
esta asignatura y las dificultades que muchos de ellos encuentran para poder compatibilizar los horarios de su centro con los ofrecidos para las enseñanzas de LANSAD. Por este motivo, se amplió la oferta de español para el nivel inicial con un nuevo grupo que se trasladó a SPIRAL, aunque con un número de plazas limitadas a 20 alumnos. Esta decisión supuso un trabajo coordinado entre el Departamento de Español y este centro de idiomas para optimizar esfuerzos y dar una respuesta conjunta a la situación.

En un primer momento, la propuesta se definió como un dispositivo de «autoformación acompañada», en el que los estudiantes tenían opción a un total de 10 horas presenciales, repartidas en talleres semanales de una hora (frente a las 24 del resto de grupos) y el acceso a todos los recursos que el centro ponía a su disposición: libros, películas, talleres, etc. Sin embargo, en un segundo momento se experimentó con las posibilidades de una formación semipresencial en las que el uso de los talleres, los recursos del centro y la plataforma pedagógica con la que cuenta la universidad se unían para dar lugar a un nuevo escenario pedagógico. Para este segundo modelo se tuvieron en cuenta experiencias anteriores similares, en las que la metodología que se seguía para un modelo de dispositivo semipresencial era el de la clase invertida (Poussard 2017). Así, el trabajo en la plataforma posibilitó a los estudiantes llevar a cabo un trabajo individual en línea, a través de materiales de diverso tipo y de tareas de comprensión oral, de práctica del vocabulario, recursos variados, etc. Por otro lado, las sesiones presenciales se usaron para la resolución de dudas y, sobre todo, para el trabajo en grupos, la realización de tareas finales y el desarrollo de la competencia oral. No cabe duda de que este tipo de dispositivo potencia la autonomía en el aprendizaje, aunque también exige una mayor capacidad de organización tanto por parte del docente como de los aprendientes. Sin embargo, el hecho de que se vehicule parte de la docencia mediante la plataforma digital permite que los encuentros presenciales se puedan explotar de otro modo.

La evaluación final a la que se sometió este grupo de alumnos fue la misma que a la que se enfrentaron el resto de grupos de español del nivel A1. En un primer momento los resultados parecen indicar una buena acogida y una respuesta favorable ante esta iniciativa, por lo que se ha continuado con ella y se ha ampliado a otros dos niveles.

\section{La clase invertida en la licenciatura de LLCER}

El método de la clase invertida también se intentó trasladar a una asignatura obligatoria que forma parte del primer año de la licenciatura de LLCER de la Universidad de Estrasburgo. En este caso, la carga semestral era de 12 horas presenciales de trabajos dirigidos, a razón de sesiones de una hora a la semana. En este caso, se podría denominar la iniciativa puesta en práctica como un «dispositivo presencial enriquecido» (Poussard 2017). El principio de clase invertida pretendía potenciar el trabajo individual y en equipo fuera del aula y permitir la explotación de las sesiones presenciales para aplicar metodologías prácticas y colaborativas por parte de los alumnos. Asimismo, se pretendía 
diversificar no solamente la tipología de tareas que se proponía a los estudiantes, sino también el soporte y los modelos de materiales de los que se disponía. En el caso de la asignatura de Historia de España, además de los recursos de creación propia, se encuentra una gran cantidad de recursos en la red para su explotación, como, por ejemplo, diversos vídeos educativos (algunos de ellos con sesiones docentes grabados por otros enseñantes de esta misma asignatura), test y Quizlet, mapas conceptuales, recursos para crear sus propias infografías, juegos, webs explicativas, etc. Podemos añadir que el uso de estos dispositivos permite programar sesiones a distancia más cortas y con menor contenido, pero de mayor asiduidad, algo que, sin duda, potencia el micro-aprendizaje.

La programación de la asignatura mediante el principio de la clase invertida posibilitó trasladar al aula otro tipo de actividades y evaluaciones docentes para las que no se hubiera contado con tiempo suficiente de no ser así.

\section{Discusión}

Las propuestas que hemos descrito en este trabajo han surgido como respuesta a los retos que hoy en día se nos presentan en el sistema universitario francés en la rama de las humanidades y de la enseñanza de lenguas extranjeras. Suponen una apuesta por el uso de las nuevas tecnologías y por la puesta en práctica de una nueva concepción de los procesos de enseñan-aprendizaje. Sin embargo, también traen aparejados nuevos desafíos, por ejemplo, ¿cómo combinamos las nociones de «espacio»y «tiempo» en nuestras secuencias didácticas?, o ¿en qué medida y cómo introducimos la enseñanza a distancia en la educación superior, tradicionalmente presencial? A su vez, ambas cuestiones nos trasladan inevitablemente a la reflexión de qué entendemos por «lugar de aprendizaje» o «entorno de aprendizaje».

Por otra parte, no queremos obviar que el uso de las nuevas tecnologías no siempre suscita un consenso sobre sus beneficios e inconvenientes, ni siquiera en el ámbito de la enseñanza llevada a cabo con adultos y jóvenes. Así, junto con las posibilidades educativas que nos ofrecen, también se les podría achacar un eventual riesgo de distracción, de dispersión o de uso inadecuado. No obstante, también parece claro que la mayor parte de nuestros estudiantes van a acabar recurriendo a algún tipo de recurso tecnológico, por lo que parece plausible que este uso se haga de forma guiada y pautada por parte del docente. Además, algunos estudios apuntan que su uso puede despertar una mayor motivación ante un público joven e, incluso, que puede potenciar la participación debido a un menor miedo al error en comparación con los entornos educativos tradicionales (Gairín Sallán y Mercader 2018). Lo que sí parece claro es que las nuevas tecnologías deben estar al servicio de la innovación docente y de las nuevas metodologías como un instrumento que las potencia, pero no deben suponer un fin en sí mismas ni son recomendables como un sustituto del trabajo docente llevado a cabo por el profesorado. 


\section{Conclusiones}

A modo de conclusión, nos gustaría manifestar que los cambios de paradigma que se vienen produciendo en el ámbito de la enseñanza-aprendizaje de lenguas extranjeras van al encuentro de aquellos que se potencian en el sistema de educación universitario e incluso en los ciclos educativos inferiores. Las consecuencias de estas transformaciones son el paso de un modelo educativo centrado en contenidos a otro que potencia la definición de competencias y objetivos para conseguir adaptarse a los cambios sociales y generacionales que se han producido en las últimas décadas. Además, se pone de manifiesto la necesidad de nuevos enfoques orientados a la acción, que se sustenten cambios metodológicos sustanciales y que ofrezcan a nuestros estudiantes un papel más activo y autónomo en los procesos de enseñanza-aprendizaje. Apostamos, además, por las puestas en práctica de nuevas metodologías docentes que trasladen el protagonismo a nuestros estudiantes y que exploren, al mismo tiempo, las posibilidades que nos ofrecen para ello las TICE.

\section{Bibliografía}

Acker P., 2017, "Posture enseignante et accompagnement du développement de l'autonomie d'apprentissage en centre de ressources de langues», Alsic, Apprentissage des langues et systèmes d'information et de communication, volumen 20, no 3, Espaces d'apprentissage et de recherche en langues à l'ère du numérique : enjeux et perspectives.

Bueno E., 2000, «De la Sociedad de la Información a la del conocimiento y el aprendizaje», IX Encuentro AECA, Gestión de la información en la Sociedad del Conocimiento y la Globalización, AECA 2000.

Chateau A., Bailly S. y Willié V., 2017, "Contribution/soutien des espaces à l'autodirection: un exemple d'hybridation du virtuel et du physique à l'université de Lorraine», Alsic, Apprentissage des langues et systèmes d'information et de communication, volumen 20, no 3 , Espaces d'apprentissage et de recherche en langues à l'ère du numérique: enjeux et perspectives.

Conseil de l'Europe, 2018, Cadre européen commun de référence pour les langues: apprendre, enseigner, évaluer. Volume complémentaire avec de nouveaux descripteurs, Estrasburgo.

Cores Bilbao E., 2017, «La mediación en el nuevo Marco Común Europeo de Referencia para las Lenguas», Marco Común Europeo de Referencia para las Lenguas. ReALL: Research in Affective Language Learning Centre.

Díaz S. C., López, L. M. y Roncallo L. L., 2017, «Entendiendo las generaciones: una revisión del concepto, clasificación y características distintivas de los Baby Boomers, X y Millennials». Clío América, no 11 (22), p. 188-204.

Gairín Sallán J. y Mercader C., 2018, «Usos y abusos de las TIC en los adolescentes», Revista de Investigación Educativa, no 36 (1), p. 125-140. 
Hamel M.J., 2017, «Portraits d'enseignants de FLS, pédagogues de l'hybride. Vers une ébauche de modèle», Alsic, Apprentissage des langues et systèmes d'information et de communication, volumen 20, no 3, Espaces d'apprentissage et de recherche en langues à l'ère du numérique: enjeux et perspectives.

Loi $\mathrm{n}^{\circ}$ 2013-595 du 8 juillet 2013 d'orientation et de programmation pour la refondation de l'école de la République.

Montero Curiel M., 2010, «El Proceso de Bolonia y las nuevas competencias», Tejuelo, no 9, p. 19-37.

Poussard C., 2017, «Quels lieux d'apprentissage en anglais Lansad en 2016? Le cas de l'université Paul Valéry Montpellier 3», Alsic, Apprentissage des langues et systèmes d'information et de communication, volumen $20, \mathrm{n}^{\circ} 3$, Espaces d'apprentissage et de recherche en langues à l'ère du numérique: enjeux et perspectives.

Socle commun de connaissances, de compétences et de culture. Décret $n^{\circ} 2015-372$ du 31-3-2015 - J.O. du 2-4-2015. Bulletin officiel de l'éducation nationale $\mathrm{n}^{\circ} 27$ du 2 juillet 2015 . 